\title{
Parallaxes of 6.7-GHz methanol masers towards the G 305.2 high-mass star formation region
}

\author{
V. Krishnan, ${ }^{1,2,3 \star}$ S. P. Ellingsen, ${ }^{2 \star}$ M. J. Reid, ${ }^{4}$ H. E. Bignall, ${ }^{3,5}$ J. McCallum, ${ }^{2}$ \\ C. J. Phillips, ${ }^{3}$ C. Reynolds ${ }^{3,5}$ and J. Stevens ${ }^{3}$ \\ ${ }^{1}$ INAF - Osservatorio Astrofisico di Arcetri, Largo E. Fermi 5, I-50125 Firenze, Italy \\ ${ }^{2}$ School of Mathematics and Physics, University of Tasmania, Private Bag 37, Hobart, Tasmania 7001, Australia \\ ${ }^{3}$ CSIRO Astronomy and Space Science, Australia Telescope National Facility, CSIRO, PO Box 76, Epping, NSW 1710, Australia \\ ${ }^{4}$ Harvard-Smithsonian Center for Astrophysics, Cambridge, Massachusetts 02138, USA \\ ${ }^{5}$ International Centre for Radio Astronomy Research, Curtin University, Building 610, 1 Turner Avenue, Bentley WA 6102, Australia
}

Accepted 2016 November 2. Received 2016 October 27; in original form 2016 August 9

\begin{abstract}
We have made measurements to determine the parallax and proper motion of the three $6.7-\mathrm{GHz}$ methanol masers G 305.200+0.019, G 305.202+0.208 and G 305.208+0.206. The combined parallax is found to be $0.25 \pm 0.05$ mas, corresponding to a distance of $4.1_{-0.7}^{+1.2} \mathrm{kpc}$. This places the G 305.2 star formation region in the Carina-Sagittarius spiral arm. The inclusion of G 305.2 increases the Galactic azimuth range of the sources in this arm by $40^{\circ}$ from Sato et al., allowing us to determine the pitch angle of this spiral with greater confidence to be $\psi=19.0$ \pm 2.6 . The first very long baseline interferometry spot maps of the $6.7-\mathrm{GHz}$ methanol masers towards these sources show that they have simple linear and ring-like structures, consistent with emission expected from class II methanol masers in general.
\end{abstract}

Key words: masers-stars: formation-Galaxy: structure.

\section{INTRODUCTION}

The Northern hemisphere very long baseline interferometry (VLBI) telescopes, including the Very Long Baseline Array, VLBI Exploration of Radio Astrometry and European VLBI Network arrays are currently involved in programmes to determine the parallaxes to high-mass star formation regions (HMSFRs) in the Milky Way, by measuring the relative separation between maser emission associated with these regions and distant background quasars. The sub-milliarcsecond (mas) accurate astrometric measurements between the maser spots and quasars allow the small trigonometric parallax signatures to be detected and the corresponding distances to these Galactic HMSF masers can be determined to an accuracy of 10 per cent at $10 \mathrm{kpc}$ (Reid \& Honma 2014).

Using over 100 parallax measurements to HMSFRs in the Milky Way, Reid et al. (2014) have determined the latest Galactic rotation and dynamical parameters, finding the circular rotation speed of the Sun to be $\Theta_{0}=240 \pm 8 \mathrm{~km} \mathrm{~s}^{-1}$ and distance to the Galactic Centre to be $R_{0}=8.34 \pm 0.16 \mathrm{kpc}$. These results have been obtained from measurements primarily from the first and second quadrants of the Galaxy and, in Reid et al. (2016), the authors demonstrate that the complete spiral structure of the Milky Way cannot be clearly distinguished without parallax distances from Southern hemisphere HMSFRs. In 2008, we initiated a project to observe $6.7-\mathrm{GHz}$

^Email: vasaantk@arcetri.astro.it (VK); Simon.Ellingsen@utas.edu.au (SPE) class II methanol masers in the Southern hemisphere using the Australian Long Baseline Array (LBA) for parallax determination.

The methanol molecule has a rich radio and millimetre wavelength spectrum (e.g. Müller, Menten \& Mäder 2004), with more than 30 observed transitions detected in interstellar space. Many of these transitions are observed to exhibit maser emission, which are empirically grouped into two classifications (Menten 1991). Class I methanol transitions are associated with distant parts of the outflows (Voronkov et al. 2006; Cyganowski et al. 2009) or other shocks (Voronkov et al. 2010) and class II masers are associated close to the young star at distances of around $10-10^{3}$ au (e.g. Ellingsen 2006; Sanna et al. 2010). The 6.7 and $12.2 \mathrm{GHz}$ class II methanol masers are two of the strongest and best-studied transitions of astrophysical maser emission with the $12.2 \mathrm{GHz}$ masers forming a complete subset of the 6.7 GHz emission (Caswell et al. 1995a; Breen et al. 2012). The $6.7 \mathrm{GHz}$ maser is a particularly important transition, as it is exclusively observed towards HMSFRs (Breen et al. 2013). Class II methanol masers are pumped by radiative excitation of the methanol molecule (Sutton et al. 2001) and are known to be strong radio sources with individual features exhibiting point-like structure even at VLBI resolution - making them excellent candidates for astrometry.

The LBA observations continue to be the only Southern hemisphere astrometric measurements of Galactic HMSFR masers for parallax determination. The first parallax distance to a southern methanol maser source has been presented in Krishnan et al. (2015) and here we provide trigonometrical parallax distances 
Table 1. A comparison of the ${ }^{13} \mathrm{CO}$ molecular clump from Hindson et al. (2013) and the 6.7-GHz methanol masers in G $305.200+0.019$, G $305.202+0.208$ and $\mathrm{G} 305.208+0.206$ (source $v_{\mathrm{lsr}}$ from Green et al. 2012). The separation column describes the angular distance between the 6.7-GHz methanol maser and the clump. G $305.21+0.21$ has a spread of $\Delta V=4.46 \mathrm{~km} \mathrm{~s}^{-1}$ in the $v_{\mathrm{lsr}}$ and a mass of $6300 \mathrm{M}_{\odot}$.

\begin{tabular}{lccc}
\hline & $\begin{array}{c}\text { Source } \\
\text { name }\end{array}$ & $\begin{array}{c}v_{\text {lsr }} \\
\left(\mathrm{km} \mathrm{s}^{-1}\right)\end{array}$ & Separation \\
\hline${ }^{13}$ CO clump: & & & \\
Masers: & G 305.21+0.21 & -43.3 & - \\
& & & \\
& G 305.200+0.019 & -33.1 & $0.2 \operatorname{arcmin}$ \\
& G 305.202+0.208 & -44.0 & 29.5 arcsec \\
& G 305.208+0.206 & -38.3 & 15.2 arcsec \\
\hline
\end{tabular}

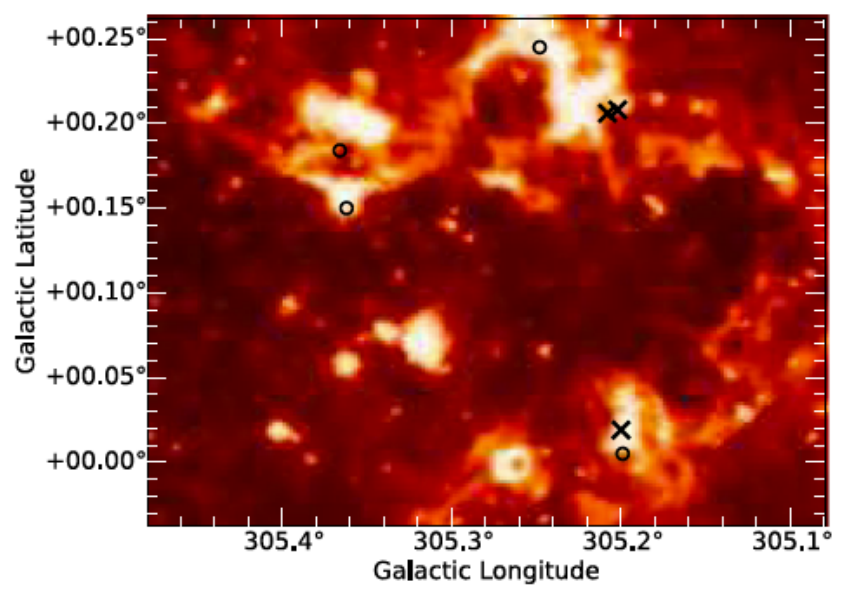

Figure 1. 6.7-GHz methanol maser emission associated with the G 305.2 HMSFR superimposed on an infrared image from Spitzer with data at 3.6 and $4.5 \mu \mathrm{m}$. The crosses indicate the positions of $\mathrm{G} 305.200+0.019$, G 305.202+0.208 and G 305.208+0.206, which were observed between 2013 and 2015 March. Positions of the other 6.7-GHz methanol masers in the vicinity from Green et al. (2012) are shown as circles.

to the $6.7-\mathrm{GHz}$ methanol maser sources in the G 305.2 star formation region.

\section{THE G 305.2 COMPLEX}

The $\mathrm{G} 305.2$ region is a vast HMSFR in the southern Galactic plane, with extensive studies undertaken by Hindson et al. (2013), Hindson et al. (2012), Hindson et al. (2010), Davies et al. (2012), Faimali et al. (2012), Walsh et al. (2007), Walsh, Lee \& Burton (2002), Walsh \& Burton (2006) and Clark \& Porter (2004) amongst others. The sources which exhibit $6.7-\mathrm{GHz}$ methanol maser emission, include G 305.200+0.019 in the south-west and G 305.202+0.208 and G 305.208+0.206 in the north-west as described in Table 1 and Fig. 1. Phillips et al. (1998) published the 6.7-GHz methanol maser spectrum and component map for G $305.202+0.208$ (as G 305.202+0.207) and report a curved distribution of maser emission with a peak of $92 \mathrm{Jy}$ at $-44.0 \mathrm{~km} \mathrm{~s}^{-1}$. Australia Telescope Compact Array (ATCA) observations by Norris et al. (1993) show G 305.208+0.206 to have a linear distribution of 6.7-GHz methanol maser spots, with a monotonic velocity gradient and a peak of $>300 \mathrm{Jy}$ at $-38.3 \mathrm{~km} \mathrm{~s}^{-1}$. The G $305.208+0.206$ site contains both $\mathrm{OH}$ and methanol masers (Caswell, Vaile \& Forster 1995b), and is located only $22 \operatorname{arcsec}$ to the east of G $305.202+0.208$. The 6.7-
GHz methanol maser emission towards G $305.200+0.019$ is weaker than the emission associated with either G $305.202+0.208$ or G 305.208+0.206, with a peak flux density of $46 \mathrm{Jy}$ at $-33.1 \mathrm{~km} \mathrm{~s}^{-1}$ (Green et al. 2012, methanol multibeam, MMB, catalogue).

Since the $1980 \mathrm{~s}$, there have been numerous attempts to determine the distance to the $\mathrm{G} 305.2$ complex using various techniques, resulting in a range of values between 2.8 and $6.2 \mathrm{kpc}$ (e.g. Danks et al. 1983; Caswell \& Haynes 1987; Walsh et al. 1997; Phillips et al. 1998; Russeil et al. 1998). The uncertainty in the derived properties of the young high-mass stars in G 305.2 is predominantly due to the range of the adopted distances by various authors. However, the discrepancy is also influenced by the observations, for example, by the arcminute-sized beams of the Infrared Astronomical Satellite (IRAS) and Parkes observations in the analysis by Walsh et al. (1997), compared to the $\sim 10$ arcsec resolution of the Walsh et al. (2007) observations. Walsh et al. (1997) use a distance of $2.8 \mathrm{kpc}$ to $\mathrm{G} 305.200+0.019$ (which they identify as G $305.202+0.019$ ) to derive a luminosity of $13.2 \times 10^{4} \mathrm{~L}_{\odot}$ for the associated IRAS source, corresponding to an $\mathrm{O} 6.5$ spectral type. In contrast, Hindson et al. (2012) use a distance of $3.8 \mathrm{kpc}$ to classify G $305.200+0.019$ as a B 1 source using the intensity of the associated UCH II region. Walsh et al. (2007) confirm the hot core nature of $\mathrm{G} 305.208+0.206$ through their molecular observations of $\mathrm{CH}_{3} \mathrm{OH}$, $\mathrm{CH}_{3} \mathrm{CN}, \mathrm{NH}_{3}, \mathrm{OCS}$ and $\mathrm{H}_{2} \mathrm{O}$, and estimate the age of the core to be between $2.0 \times 10^{4}$ and $1.5 \times 10^{5}$ yr. Unlike G 305.202+0.208 (referred to as G 305B by Walsh et al. 2001), which is associated with a very bright and reddened IR source (Walsh et al. 1999; De Buizer 2003), there is little evidence to suggest coincident IR emission associated with G 305.208+0.206 (referred to as G 305A by Walsh et al. 2001). Based on the absence of detectable $\mathrm{H}$ II regions, Walsh \& Burton (2006) propose that G 305.202+0.208 and G $305.208+0.206$ are at early stages of stellar evolution, with G $305.208+0.206$ likely to be the younger source, as it shows strong millimeter-continuum emission (Hill et al. 2005; Csengeri et al. 2014) and no detectable IR emission. Assuming a distance of $6.2 \mathrm{kpc}$, Phillips et al. (1998) propose upper-limit spectral types of $<$ B 1 and $<$ B 0.5 for G 305.208+0.206 and G 305.202+0.208, derived from an absence of associated UC $\mathrm{H}$ II regions (with a detection limit of $\sim 0.5 \mathrm{mJy}$ beam $^{-1}$ at $8.6-\mathrm{GHz}$ ).

Table 1 describes the properties of the most massive ${ }^{13} \mathrm{CO}$ molecular clump from Hindson et al. (2013), which is associated with G 305.200+0.019, G 305.202+0.208 and G 305.208+0.206. Using Galactic parameter values of $\Theta_{0}=240 \mathrm{~km} \mathrm{~s}^{-1}$ (including an uncertainty of $8 \mathrm{~km} \mathrm{~s}^{-1}$ ) and $R_{0}=8.34 \mathrm{kpc}$ (Reid et al. 2014), we find the (near) kinematic distance to the $\mathrm{G} 305.2$ region to be $4.3_{-1.4}^{+2.2} \mathrm{kpc}$. A known caveat of the kinematic distance technique is that it is susceptible to large error if the sources exhibit anomalous peculiar motions (e.g. Xu et al. 2009). Table 1 shows that there is a difference of $\sim 10 \mathrm{~km} \mathrm{~s}^{-1}$ between the MMB catalogue peak velocity of the $6.7-\mathrm{GHz}$ methanol maser emission and the associated ${ }^{13} \mathrm{CO}$ clump G $305.21+0.21$. Therefore, using the kinematic distance technique can produce results with considerable error for the maser sources in this region.

As a prominent southern star formation region, improved accuracy in the distance determination to the $\mathrm{G} 305.2$ complex is clearly significant and here we report the trigonometric parallax distance to this complex.

\section{OBSERVATIONS}

Five epochs of phase-referenced observations (2013 March, June, August, November and 2015 March) were made of the $6.7-\mathrm{GHz}$ 
Table 2. Phase-referenced 6.7-GHz methanol maser observations between 2013 and 2015 March including the start time and the duration of the observations. The participating stations are the Australia Telescope Compact Array (AT), Ceduna (CD), Hartebeesthoek (HH), Hobart (HO), Mopra (MP), Parkes (PA) and Warkworth (WA).

\begin{tabular}{lccccc}
\hline Year & Date & DOY & $\begin{array}{c}\text { UT } \\
\text { start }\end{array}$ & $\begin{array}{c}\text { Duration } \\
(\mathrm{h})\end{array}$ & $\begin{array}{c}\text { Participating } \\
\text { stations }\end{array}$ \\
\hline 2013 & March 18 & 077 & $04: 00$ & 24 & AT, CD, HH, HO, PA \\
2013 & June 17 & 168 & $02: 30$ & 19.5 & AT, CD, HH, HO, MP, PA \\
2013 & August 14 & 226 & $18: 00$ & 24 & AT, CD, HH, HO, MP, PA \\
2013 & November 19 & 323 & $12: 00$ & 23 & AT, CD, HO, MP, PA \\
2015 & March 27 & 086 & $02: 00$ & 24 & AT, CD, HH, HO, MP, PA, WA \\
\hline
\end{tabular}

methanol masers in G 305.200+0.019, G 305.202+0.208 and G 305.208+0.206, as well as of the associated quasars $\mathrm{J}$ 1254-6111, J 1256-6449 and J 1312-6035 using the LBA. The $\mathrm{J} 2000$ coordinates, which we used for the maser observations, were obtained from the MMB (Green et al. 2012) for G 305.200+0.019 at $\alpha=13^{\mathrm{h}} 11^{\mathrm{m}} 16.93, \delta=-62^{\circ} 45^{\prime} 55^{\prime \prime}$. 1 , G $305.202+0.208$ at $\alpha=13^{\mathrm{h}} 11^{\mathrm{m}} 10^{\varsigma} 49, \delta=-62^{\circ} 34^{\prime} 38^{\prime \prime} .8$ and G $305.208+0.206$ at $\alpha=$ $13^{\mathrm{h}} 11^{\mathrm{m}} 13^{\mathrm{s}} .71, \delta=-62^{\circ} 34^{\prime} 41^{\prime \prime} .4$. The latter two sources are separated by 22 arcsec and we pointed the telescopes mid-way between the two source positions for the observations, but correlated the individual sources at the respective MMB positions. The primary beams of the individual telescopes are greater than 1 arcmin at $6.7-\mathrm{GHz}$ and so there is little loss in sensitivity in employing this procedure. Table 3 contains updated coordinates for these sources, based on the absolute position of J 1254-6111 (see Section 4). The coordinates for J 1254-6111 and J 1256-6449 in Table 3 are from Petrov et al. (2011) with positional uncertainties of 1.49 and 1.26 mas, respectively. The coordinates which were used for J 1312-6035 were $\alpha=13^{\mathrm{h}} 12^{\mathrm{m}} 12^{\mathrm{s}} \cdot 34, \delta=-60^{\circ} 35^{\prime} 38^{\prime \prime} .1$ and are reported to be accurate to $1^{\prime \prime}$ (Murphy et al. 2010). We present updated coordinates for this source in Table 3 and used these in our analyses.

Observations typically lasted for $\lesssim 24$ h (Table 2 ), with approximately one-third of the time used for observations of the 6.7$\mathrm{GHz}$ methanol masers and associated background quasars. The phase-referencing technique involved alternating scans for 2 min on the target maser with scans lasting $2 \mathrm{~min}$ on the nearby $\left(\sim 2^{\circ}\right)$ quasars. We scheduled our observations to ensure that J 1254-6111, J 1256-6449 and J 1312-6035 were phase-referenced to all maser sources.

The phase-referenced observations were interspersed with tropospheric calibration observations which consisted of short (2 min per source) scans of 12-18 quasars from the International Celestial Reference Frame (ICRF) Second Realization catalogue (Ma et al. 2009). The ICRF sources were observed over as broad an azimuth range (generally at low elevation) and were arranged into 45 min blocks with intervals of 3-6 h between consecutive blocks.

The data were correlated at Curtin University using the DIFX ${ }^{1}$ software (Deller et al. 2011). We correlated a $2 \mathrm{MHz}$ zoom-band with 2048 channels for the maser data, giving spectral channel width of $0.977 \mathrm{kHz}$ and corresponding velocity separation of $0.055 \mathrm{~km} \mathrm{~s}^{-1}$. Only a fraction of the recorded $32 \mathrm{MHz}$ was used because the maser emission covers a small bandwidth. In contrast, we correlated the full recorded bandwidth for the phase quasar observations. In the 2013 March epoch, 256 spectral channels were used per

\footnotetext{
${ }^{1}$ This work made use of the Swinburne University of Technology software correlator, developed as part of the Australian Major National Research Facilities Programme and operated under licence.
}

$16 \mathrm{MHz}$ bandwidth. In the remaining epochs, 32 spectral channels were used per $16 \mathrm{MHz}$ bandwidth corresponding to resolutions of 62.5 and $500 \mathrm{kHz}$, respectively. We used identical correlation parameters for the ICRF and background quasar data for each epoch.

The full details of the observations including the LBA setup, correlation parameters and calibration procedures can be found in Krishnan et al. (2015).

\section{DATA CALIBRATION}

We followed the standard data reduction pathway for VLBI data calibration of the ICRF and phase-referencing mode observations using the Astronomical Image Processing System (Greisen 2003).

We removed the estimated ionospheric delay (determined from global models based on GPS total electron content observations; Walker \& Chatterjee 1999), the Earth Orientation Parameters (EOPs), parallactic angle effects and clock drift for each observatory from the ICRF multiband delays before correcting for Doppler effects which manifest as a spectral frequency shift. Fig. 2 shows the autocorrelation spectra of the 6.7-GHz methanol maser emission associated with G 305.199+0.005, G 305.200+0.019, G 305.202+0.208 and G 305.208+0.206. The local standard of rest velocities $\left(v_{\mathrm{lsr}}\right)$ of $-42.8,-33.1,-44.0$ and $-38.3 \mathrm{~km} \mathrm{~s}^{-1}$ correspond to the maser spectral peaks of the positions reported by Green et al. (2012). We performed amplitude calibration using ACCOR to correct imperfect sampler statistics during correlation and ACFIT to scale the spectra at all observing stations from a single autocorrelation scan of each maser source.

Delay calibration was performed on the phas-reference quasar data set using J 1254-6111, which was the brightest quasar with the most accurate initial position. We applied the delay solutions (with zeroed rates) to the other phase-reference quasars and employed the procedure in Krishnan et al. (2015) to apply the corrections to the maser data set.

Due to the relative strength of the maser emission compared to the quasars (see Tables 4 and 5), we treated the masers as the calibrator and the quasar as the target for phase-referencing. In order to select the best maser features for astrometry, we studied the crosscorrelation spectra and identified the maser features which showed the least flux variations across all baselines and epochs. These were found to be the $-33.1 \mathrm{~km} \mathrm{~s}^{-1}$ peak for $\mathrm{G} 305.200+0.019$, the $-44.0 \mathrm{~km} \mathrm{~s}^{-1}$ peak for $\mathrm{G} 305.202+0.208$ and the $-38.3 \mathrm{~km} \mathrm{~s}^{-1}$ peak for $\mathrm{G} 305.208+0.206$ (Fig. 2). We then used the associated maser spectral channel for phase calibration, and produced images of the emission, finding them to be point-like and persistent across all epochs. This demonstrated that they were suitable for astrometry and we transferred the phase solutions to J 1254-6111, 

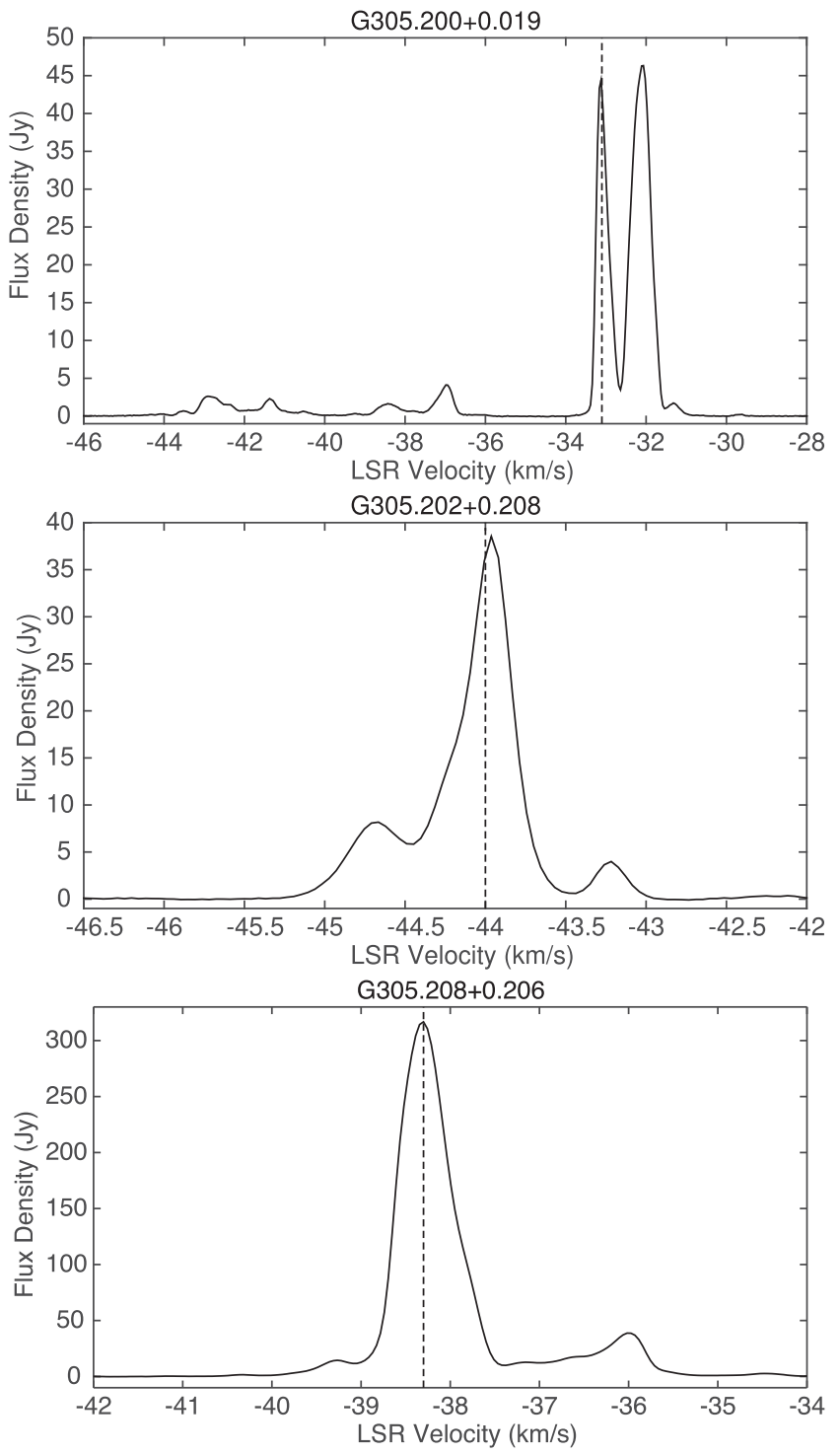

Figure 2. The autocorrelation spectra using all antennas from the 2013 November session. Top: G $305.199+0.005\left(-45.0\right.$ to $\left.-40.0 \mathrm{~km} \mathrm{~s}^{-1}\right)$ and G $305.200+0.019\left(-38.0\right.$ to $\left.-29.5 \mathrm{~km} \mathrm{~s}^{-1}\right)$; the emission in the region with peak at $-38.5 \mathrm{~km} \mathrm{~s}^{-1}$ was not detected in Green et al. (2012) (see Section 6.3.1), middle: G 305.202+0.208 and bottom: G 305.208+0.206. The dashed vertical lines indicate the channel which we used for astrometry.

J 1256-6035 and J 1312-6035 to complete the phase-reference procedure.

We averaged all channels for each phase-reference quasar and produced images of the emission using a Gaussian restoring beam of $\sim 4.5$ mas $^{2}$ (averaged over all sources and epochs) and report detections for J 1254-6111, J 1256-6035 and J 1312-6035 on VLBI baselines. We also observed J 1259-6519 (McConnell et al. 2012) for $6 \mathrm{~min}$ to test its suitability as a quasar for phase-referencing in the 2013 March epoch. We failed to detect it at an upper limit for detection at five times the image rms (from a box of size $1.5 \operatorname{arcsec}^{2}$ ) and hence excluded it from subsequent observations. The detected quasars appear to be dominated by single components, showing deviation from point-like structure at levels $<10$ per cent of the peak flux density (Fig. 3). Residual phase errors contribute to distortions in the final images. We located the centroid position of the quasar by fitting a 2 D Gaussian to the deconvolved quasar emission using

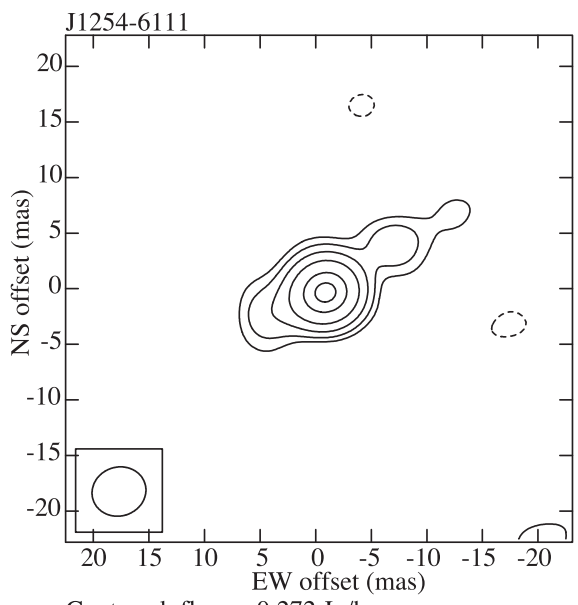

Cont peak flux $=0.272 \mathrm{Jy} /$ beam

$\%$ Levs $=0.272 *(-90,-64,-32,-16,-8,-4$, $4,8,16,32,64,90)$

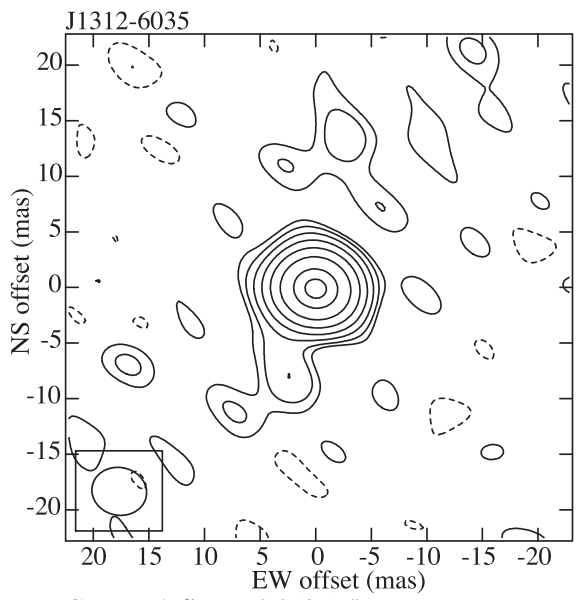

Cont peak flux $=0.019 \mathrm{Jy} /$ beam

$\%$ Levs $=0.019 *(-90,-64,-32,-16,-8,-4,-2$,

$-1,1,2,4,8,16,32,64,90)$

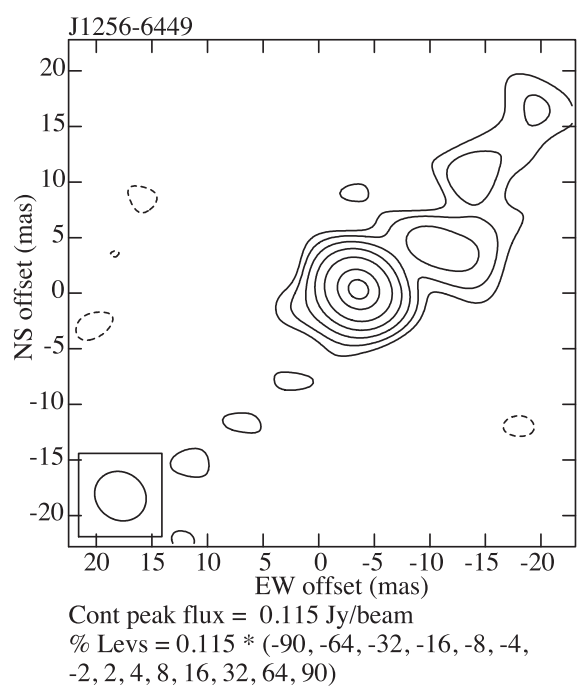

Figure 3. J 1254-6111 (top), J 1312-6035 (middle) and J 1256-6035 (bottom) phase-referenced to G $305.202+0.208$ from the 2013 June session. The quasars showed consistent centroid structure dominated by a single peak throughout all epochs. There was some variability in the quality of the images from one epoch to another, with image distortions resulting from residual phase errors. However, these do not prevent us from accurately measuring the position of the core. 
Table 3. Coordinates of the observed sources between 2013 and 2015 March. The separation and position angle columns describe the offset in the sky between the respective quasar and the $6.7-\mathrm{GHz}$ methanol masers in $\mathrm{G} \mathrm{305.200+0.019}$. The offset and separation of the methanol masers in G 305.202+0.208 and G 305.208+0.206 with respect to G 305.200+0.019 is also listed. The reported positions for G 305.200+0.019, G 305.202+0.208, G $305.208+0.206$ and J 1312-6035 are revised based on the 2013 June epoch (see Section 4).

\begin{tabular}{|c|c|c|c|c|c|}
\hline & $\begin{array}{c}\text { Source } \\
\text { name }\end{array}$ & $\begin{array}{c}\text { Separation } \\
\left(^{\circ}\right)\end{array}$ & $\begin{array}{c}\text { Position } \\
\text { angle } \\
\left(^{\circ}\right)\end{array}$ & $\begin{array}{l}\text { RA } \\
\left({ }^{\mathrm{hms}}\right)\end{array}$ & $\begin{array}{l}\text { Dec. } \\
\left(\circ^{\prime \prime \prime}\right)\end{array}$ \\
\hline \multicolumn{6}{|l|}{ Masers: } \\
\hline & G $305.200+0.019$ & - & - & 131116.8912 & -624555.008 \\
\hline & G $305.202+0.208$ & 0.19 & 356.24 & 131110.4904 & -623438.856 \\
\hline & G $305.208+0.206$ & 0.19 & 358.12 & 131113.7017 & -623441.397 \\
\hline \multicolumn{6}{|l|}{ Detected quasars: } \\
\hline & J $1254-6111$ & 2.50 & 307.21 & 125446.5768 & -611134.969 \\
\hline & J 1256-6449 & 2.65 & 217.58 & 125603.4030 & -644914.817 \\
\hline & J $1312-6035$ & 2.17 & 2.99 & 131212.2928 & -603538.220 \\
\hline \multicolumn{6}{|l|}{ Non-detected quasar: } \\
\hline & J $1259-6519$ & 2.88 & 205.54 & 125923.9000 & -651953.200 \\
\hline
\end{tabular}

JMFIT and recorded the offset of the emission peak from the centre of the image field for all epochs. We then reversed the signs of the offsets in order to represent the shift of the maser emission with respect to the quasar. These offsets are presented in Tables 4 and 5 for G $305.200+0.019$ and G 305.202+0.208, respectively. The errors in the fitted positions for the offset positions in Tables 4 and 5 are from JMFIT and are in agreement with theoretical predictions of astrometric accuracy to within $\sim 0.02$ mas.

The input source position errors are $\sim 0.4$ arcsec for the MMB coordinates for all the maser sources and better than 2 mas for two of the phase-reference quasars. Hence, for these quasars, any offset of the image from the centre of the field is due to the offset of the correlated maser position from the true maser position. We refined the coordinates for the maser feature used for phase-referencing by iteratively adjusting them for the 2013 June epoch. We repeated this until the J 1254-6111 emission was at the centre of the image, post-phase-referencing to the maser. We chose J 1254-6111 for this process as it was the brightest of the quasars with the best determined position. We then used the updated maser coordinates for all other epochs. As the phase solutions are derived assuming that the maser is at a given position, accurate coordinates that are essential as positional errors produce residual phase shifts which cannot be perfectly modelled as a position shift alone when applied to the quasar. This will result in severe image degradation in the weak quasar sources (Beasley \& Conway 1995; Reid et al. 2009a). From our phase-referenced observations, we present updated coordinates for G 305.200+0.019, G $305.202+0.208$ and $G 305.208+0.206$ in Table 3. The offset between the source coordinates in Table 3 and Green et al. (2012) for G $305.200+0.019$ is 0.282 arcsec , G 305.202+0.208 is 0.057 arcsec and G $305.208+0.206$ is 0.076 arcsec. We also present updated coordinates for J $1312-6035$ to better than 2 mas in Table 3, with an offset of 0.368 arcsec from the Murphy et al. (2010) position.

The change in the position of the maser feature used for astrometry was modelled independently in right ascension and declination and included corrections for the ellipticity of Earth's orbit (Reid et al. 2014). We assigned a priori astrometric uncertainties in right ascension and declination to account for systematic uncertainties in each coordinate. These estimates were iteratively adjusted until the $\chi_{v}^{2}$ value per degree of freedom of $\sim 1$ was obtained for the parallax model. These uncertainties were added in quadrature to the formal errors of the offsets in Tables 4 and 5 to obtain the parallax which we present in the following section.

We have not included the data obtained from the Hartebeesthoek $26 \mathrm{~m}$ and Warkworth $30 \mathrm{~m}$ antennas for the results presented in this paper. The location of the Hartebeesthoek antenna with respect to the rest of the LBA affects its participation in the ICRF observations, preventing us from correcting the clock rate at this observatory. We are currently exploring alternative methods to determine the clock rate from Hartebeesthoek. The Warkworth antenna was included in the array on a test basis for the first time for maser astrometry in 2015 March (see Table 2). However, we were unable to obtain sufficient valid data from this antenna using our current calibration pipeline. We anticipate that astrometric results from measurements including Warkworth will be presented in the future as these issues are resolved.

\section{PARALLAX MEASUREMENTS}

The parallax of G $305.200+0.019$ is measured to be $0.21 \pm 0.06$ mas (Table 4 and Fig. 4) and is a variance-weighted average derived from measurements with respect to J 1254-6111, J 1312-6035 and J 1256-6449. The variance weighted average parallax of G $305.202+0.208$ is measured to be $0.42 \pm 0.13$ mas (Table 5 and Fig. 5) with respect to J 1254-6111, J 1312-6035 and J 1256-6449. The sources are almost certainly contained within the same giant molecular cloud and so we have averaged the values to present an estimated parallax to the $\mathrm{G} 305.2$ region in general. Due to the large uncertainty in the G 305.202+0.208 measurement, we have adopted a variance-weighted approach to obtain a parallax of $0.25 \pm 0.05$ mas, corresponding to $4.1_{-0.7}^{+1.2} \mathrm{kpc}$, which we present as the current best estimate of the distance to the $\mathrm{G} 305.2$ region in Table 6.

In order to constrain errors in the measured proper motions, we made image cubes of the maser emission and analysed the changes in the spot distribution from 2013 to 2015 March. We added the median of the spot motion distribution to the modelled proper motion $\left(\mu_{x}, \mu_{y}\right)$ of the $-33.1 \mathrm{~km} \mathrm{~s}^{-1}$ feature in $\mathrm{G} 305.200+0.019$ and of the $-44.0 \mathrm{~km} \mathrm{~s}^{-1}$ feature in $\mathrm{G} 305.202+0.208$ and included half of the spread from the respective distributions to the formal model errors. This was done to account for determining the motion of the HMSFR region from a single maser spot. We report the proper motion of the $-33.1 \mathrm{~km} \mathrm{~s}^{-1}$ feature in $\mathrm{G} 305.200+0.019$ with errors

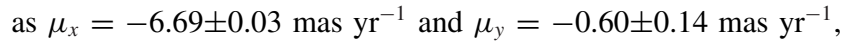


Table 4. The differential fitted positions of the $-33.1 \mathrm{~km} \mathrm{~s}^{-1}$ feature in $\mathrm{G} 305.200+0.019$ with respect to J 1254-6111, J 1312-6035 and J $1256-6449$ and the corresponding parallax. The flux density of the maser peak emission corresponding to the $-33.1 \mathrm{~km} \mathrm{~s}^{-1}$ feature is also presented. We used an emission-free channel to obtain the maser rms. The listed rms for all images have been obtained using the histogram in IMEAN.

\begin{tabular}{|c|c|c|c|c|c|c|c|c|c|c|}
\hline Source & Epoch & $\begin{array}{l}x \text { offset } \\
\quad \text { (mas) }\end{array}$ & Error & $\begin{array}{l}y \text { offset } \\
\text { (mas) }\end{array}$ & Error & $\begin{array}{l}\text { Quasar } \\
\text { flux density } \\
\qquad(\mathrm{mJy})\end{array}$ & $\mathrm{rms}$ & $\begin{array}{r}\text { G } 305.200+0 \\
\text { Flux density } \\
\text { (Jy) }\end{array}$ & 9 & $\begin{array}{l}\text { Parallax } \\
\text { (mas) }\end{array}$ \\
\hline \multirow[t]{5}{*}{ J $1254-6111$} & 2013.210 & 2.362 & 0.056 & -0.372 & 0.032 & 172.6 & 1.7 & 28.34 & 0.02 & $0.20 \pm 0.08$ \\
\hline & 2013.460 & 0.381 & 0.007 & -0.235 & 0.009 & 302.3 & 1.2 & 28.56 & 0.02 & \\
\hline & 2013.621 & -0.793 & 0.023 & -0.136 & 0.017 & 159.7 & 1.2 & 28.00 & 0.02 & \\
\hline & 2013.887 & -2.335 & 0.021 & 0.001 & 0.021 & 159.2 & 3.6 & 25.46 & 0.02 & \\
\hline & 2015.236 & -11.479 & 0.020 & -0.665 & 0.014 & 183.1 & 1.2 & 28.43 & 0.03 & \\
\hline \multirow[t]{5}{*}{ J 1312-6035 } & 2013.210 & 1.398 & 0.036 & 0.424 & 0.018 & 18.8 & 2.3 & & & $0.21 \pm 0.09$ \\
\hline & 2013.460 & -0.533 & 0.031 & 0.694 & 0.040 & 14.8 & 2.7 & & & \\
\hline & 2013.621 & -1.789 & 0.023 & -0.317 & 0.019 & 17.5 & 1.8 & & & \\
\hline & 2013.887 & -3.228 & 0.103 & -0.981 & 0.099 & 5.7 & 2.3 & & & \\
\hline & 2015.236 & -12.066 & 0.041 & -1.327 & 0.029 & 17.3 & 2.5 & & & \\
\hline \multirow[t]{5}{*}{ J $1256-6449$} & 2013.210 & 3.988 & 0.011 & -1.808 & 0.009 & 87.7 & 1.2 & & & $0.40 \pm 0.33$ \\
\hline & 2013.460 & 3.012 & 0.018 & -0.564 & 0.022 & 81.4 & 8.6 & & & \\
\hline & 2013.621 & 2.359 & 0.039 & -0.987 & 0.026 & 56.9 & 8.8 & & & \\
\hline & 2013.887 & 1.815 & 0.071 & -1.035 & 0.066 & 26.4 & 1.5 & & & \\
\hline & 2015.236 & -9.062 & 0.012 & -1.292 & 0.009 & 63.6 & 3.1 & & & \\
\hline
\end{tabular}

Table 5. The differential fitted positions of the $-44.0 \mathrm{~km} \mathrm{~s}^{-1}$ feature in $\mathrm{G} 305.202+0.208$ with respect to J $1254-6111$, J $1256-6449$ and J $1312-6035$ and the corresponding parallax. The flux density of the maser peak emission corresponding to the $-44.0 \mathrm{~km} \mathrm{~s}^{-1}$ feature is also presented. We used an emission-free channel to obtain the maser rms. The listed rms for all images have been obtained using the histogram in IMEAN.

\begin{tabular}{|c|c|c|c|c|c|c|c|c|c|c|}
\hline Source & Epoch & $\begin{array}{l}x \text { offset } \\
\quad \text { (mas) }\end{array}$ & Error & $\begin{array}{l}y \text { offset } \\
\text { (mas }\end{array}$ & Error & $\begin{array}{l}\text { Quasar } \\
\text { Flux density } \\
\qquad(\mathrm{mJy})\end{array}$ & rms & $\begin{array}{r}\text { G } 305.202+ \\
\text { flux density } \\
\text { (Jy) }\end{array}$ & $\begin{array}{l}08 \\
\text { rms }\end{array}$ & $\begin{array}{l}\text { Parallax } \\
\text { (mas) }\end{array}$ \\
\hline \multirow[t]{5}{*}{ J 1254-6111 } & 2013.210 & 3.240 & 0.034 & 0.583 & 0.023 & 195.1 & 4.3 & 20.56 & 0.02 & $0.36 \pm 0.22$ \\
\hline & 2013.460 & 0.865 & 0.022 & 0.324 & 0.021 & 273.3 & 3.0 & 26.60 & 0.03 & \\
\hline & 2013.621 & -0.604 & 0.024 & 0.478 & 0.021 & 174.7 & 2.7 & 22.08 & 0.02 & \\
\hline & 2013.887 & -1.462 & 0.047 & 0.127 & 0.047 & 236.3 & 6.8 & 19.86 & 0.02 & \\
\hline & 2015.236 & -11.068 & 0.033 & -0.229 & 0.030 & 114.5 & 1.7 & 16.31 & 0.07 & \\
\hline \multirow[t]{5}{*}{ J 1312-6035 } & 2013.210 & 2.508 & 0.029 & 0.959 & 0.021 & 16.7 & 2.8 & & & $0.21 \pm 0.22$ \\
\hline & 2013.460 & -0.253 & 0.021 & 0.124 & 0.018 & 19.7 & 1.8 & & & \\
\hline & 2013.621 & -1.529 & 0.051 & 0.259 & 0.048 & 11.0 & 2.9 & & & \\
\hline & 2013.887 & -2.740 & 0.038 & -0.368 & 0.033 & 10.8 & 4.3 & & & \\
\hline & 2015.236 & -11.947 & 0.062 & -1.507 & 0.059 & 17.4 & 4.7 & & & \\
\hline \multirow[t]{5}{*}{ J 1256-6449 } & 2013.210 & 6.775 & 0.082 & -0.291 & 0.046 & 39.5 & 1.7 & & & $0.68 \pm 0.23$ \\
\hline & 2013.460 & 3.611 & 0.018 & -0.620 & 0.016 & 128.4 & 1.2 & & & \\
\hline & 2013.621 & 2.352 & 0.040 & -0.487 & 0.036 & 54.1 & 1.4 & & & \\
\hline & 2013.887 & 2.078 & 0.200 & -0.555 & 0.154 & 30.1 & 2.8 & & & \\
\hline & 2015.236 & -8.689 & 0.064 & -0.979 & 0.065 & 52.4 & 1.8 & & & \\
\hline
\end{tabular}

corresponding to -130.0 and $-11.7 \mathrm{~km} \mathrm{~s}^{-1}$ at a distance of $4.1 \mathrm{kpc}$. The proper motion of the $-44.0 \mathrm{~km} \mathrm{~s}^{-1}$ feature in $\mathrm{G} 305.202+0.208$

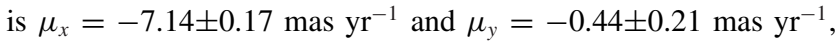
corresponding to -138.8 and $-8.6 \mathrm{~km} \mathrm{~s}^{-1}$ at a distance of $4.1 \mathrm{kpc}$.

The uncertainties in $\left(\mu_{x}, \mu_{y}\right)$ for both sources correspond to internal motions of $\lesssim 10 \mathrm{~km} \mathrm{~s}^{-1}$ in the maser emission and are consistent with proper motion estimates of 6.7-GHz methanol masers in HMSFRs (e.g. Goddi, Moscadelli \& Sanna 2011; Sugiyama et al. 2014; Moscadelli \& Goddi 2014). A more detailed analysis of the internal motions of the $6.7-\mathrm{GHz}$ emission in the $\mathrm{G} 305.2$ sources is beyond the scope of the current paper and will be the subject of future analysis.

We were unable to determine the parallax of G 305.208+0.206 from our observations. It is possible that the observed blending of the emission in contiguous channels of the strongest features at
$-38 \mathrm{~km} \mathrm{~s}^{-1}$ and around $-36 \mathrm{~km} \mathrm{~s}^{-1}$ (see Section 6.3.2) undermines the assumption that the emission is point-like at VLBI resolution and indicates that this is a source which may be unsuitable for sub-milliarcsecond astrometry for the purposes of parallax determination.

The dominant source of errors in phase-referencing at observations $>5 \mathrm{GHz}$, comes from the clocks and unmodelled troposphere (Mioduszewski \& Kogan 2009). Krishnan et al. (2015) examined how the difficulties in effectively correcting for ionospheric phase can affect the multiband delay solutions from the ICRF observations for the LBA. We found an rms noise of $\sim 0.1 \mathrm{nsec}$ in the multiband delays from the ICRF observations, which can correspond to large path lengths of tens of centimeters. We therefore applied the linear clock drift rate from the multiband delays to the phase-referenced data and omitted the zenith atmospheric delay corrections during 

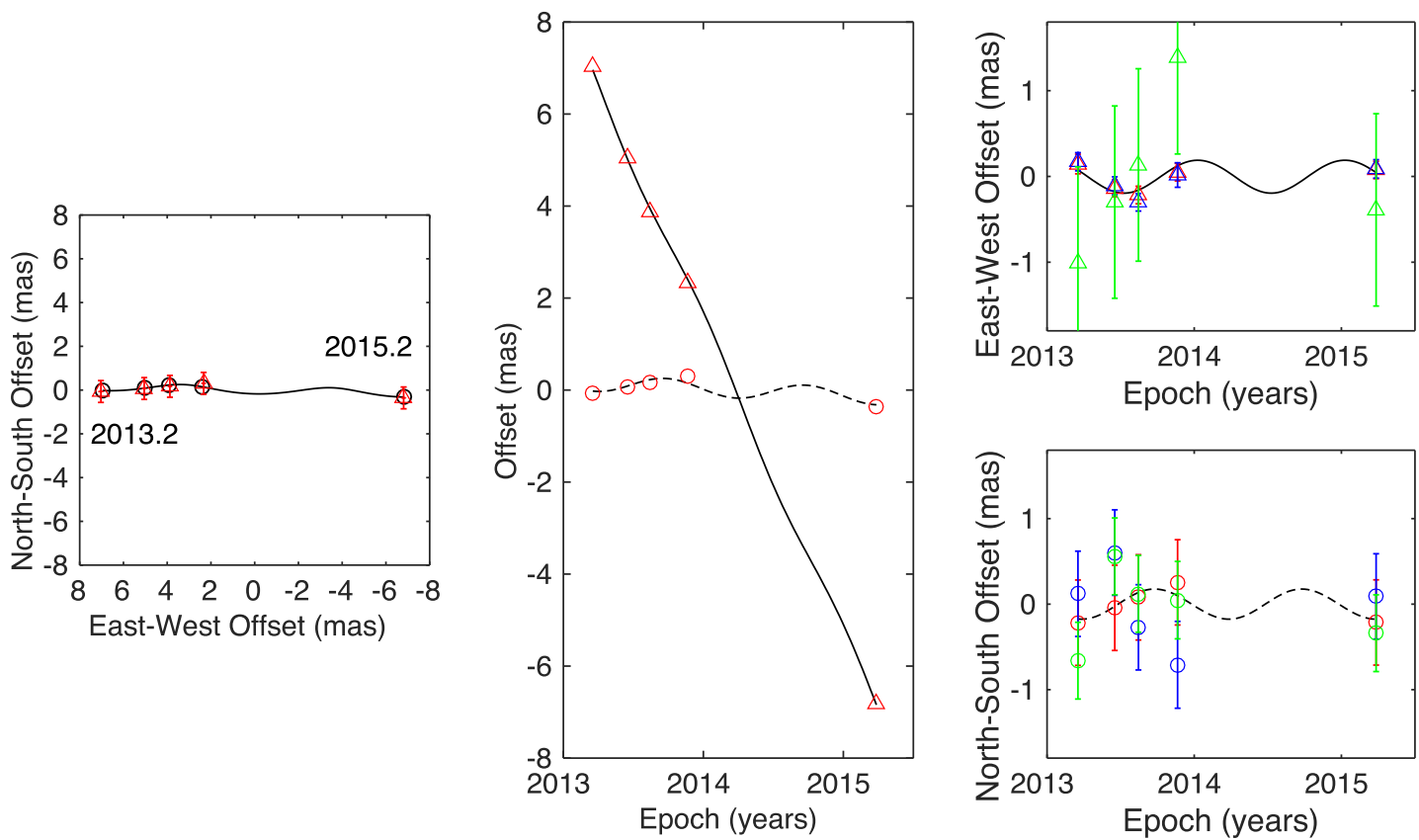

Figure 4. Parallax and proper motion of the $-33.1 \mathrm{~km} \mathrm{~s}^{-1}$ reference feature in G $305.200+0.019$ with respect to J $1254-6111$ (red), J $1312-6035$ (blue) and J 1256-6449 (green). Left-hand panel: the sky positions with the first and last epochs labelled. We have opted to show the positions with respect to a single quasar for clarity. The expected positions from the fits are indicated with black circular markers. Middle panel: east-west (triangles) and north-south (circles) motion of the position offsets and best-combined parallax and proper motion fits versus time. Right-hand panels: the east-west (top) and north-south (bottom) parallax signature with the best-fitting proper motions removed.
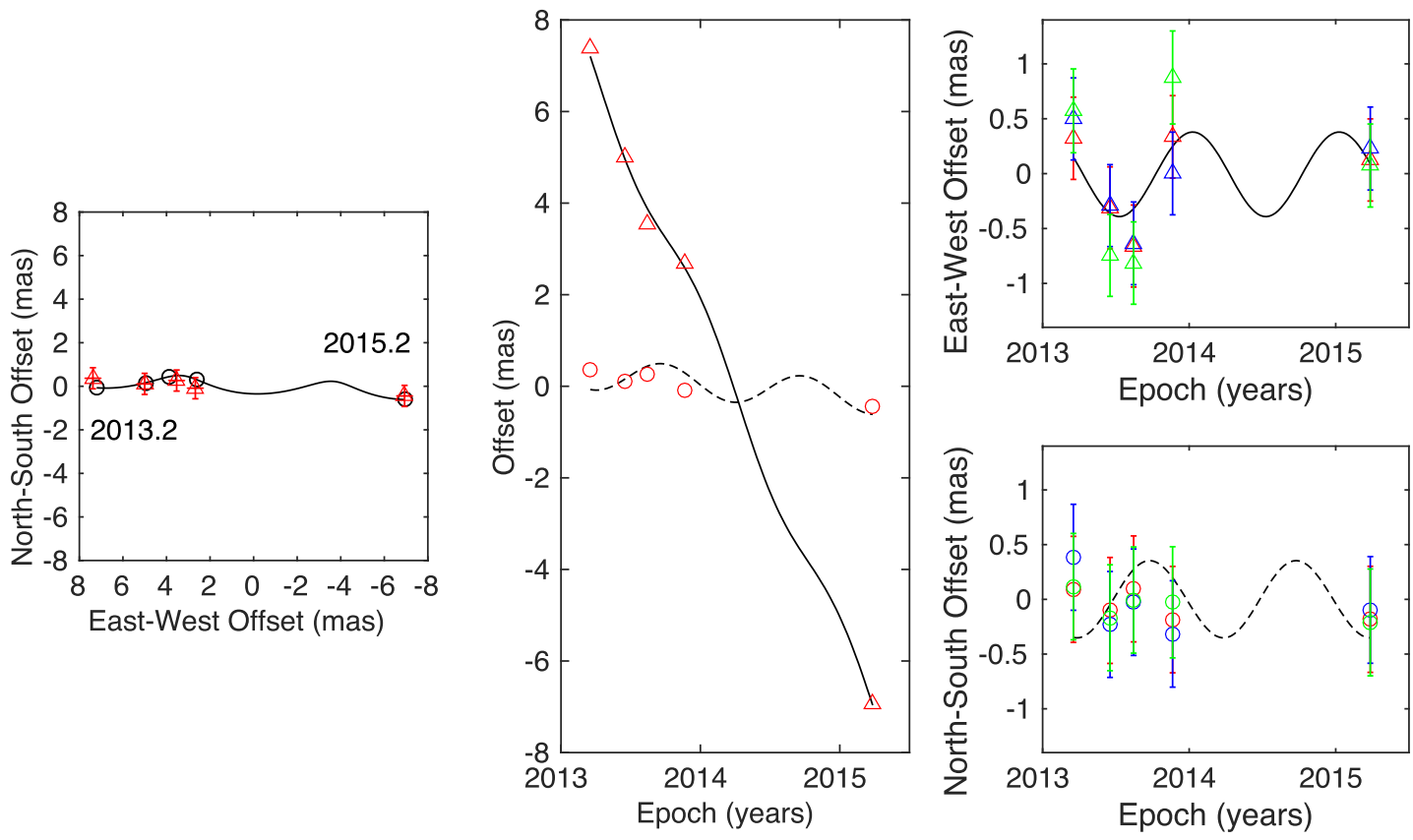

Figure 5. Parallax and proper motion of the $-44.0 \mathrm{~km} \mathrm{~s}^{-1}$ reference feature in G $305.202+0.208$ with respect to J $1254-6111$ (red), J $1312-6035$ (blue) and J 1256-6449 (green). Left-hand panel: the sky positions with the first and last epochs labelled. We have opted to show the positions with respect to a single quasar for clarity. The expected positions from the fits are indicated with black circular markers. Middle panel: east-west (triangles) and north-south (circles) motion of the position offsets and best-combined parallax and proper-motion fits versus time. Right-hand panels: the east-west (top) and north-south (bottom) parallax signature with the best-fitting proper motions removed. 
Table 6. Summary of the parallax, distance and proper motion measurements of G $305.200+0.019$ and G 305.202+0.208.

\begin{tabular}{lccc}
\hline $\begin{array}{l}\text { Source } \\
\text { name }\end{array}$ & $\begin{array}{c}\text { Parallax } \\
\text { (mas) }\end{array}$ & $\begin{array}{c}\mu_{x} \\
\left(\mathrm{mas} \mathrm{yr}^{-1}\right)\end{array}$ & $\begin{array}{c}\mu_{y} \\
\left(\mathrm{mas} \mathrm{yr}^{-1}\right)\end{array}$ \\
\hline G 305.200+0.019 & $0.21 \pm 0.06$ & $-6.69 \pm 0.03$ & $-0.60 \pm 0.14$ \\
G 305.202+0.208 & $0.42 \pm 0.13$ & $-7.14 \pm 0.17$ & $-0.44 \pm 0.21$ \\
Variance-weighted average: & $0.25 \pm 0.05$ & & \\
Corresponding distance: & $4.1_{-0.7}^{+1.2} \mathrm{kpc}$ & & \\
\hline
\end{tabular}

Table 7. Peculiar motions in a reference frame that is rotating with the Galaxy.

\begin{tabular}{lcccr}
\hline $\begin{array}{l}\text { Source } \\
\text { name }\end{array}$ & $\begin{array}{c}\mathrm{D} \\
(\mathrm{kpc})\end{array}$ & $\begin{array}{c}U \\
\left(\mathrm{~km} \mathrm{~s}^{-1}\right)\end{array}$ & \multicolumn{1}{c}{$\begin{array}{c}V \\
\left(\mathrm{~km} \mathrm{~s}^{-1}\right)\end{array}$} & \multicolumn{1}{c}{$\begin{array}{c}W \\
\left(\mathrm{~km} \mathrm{~s}^{-1}\right)\end{array}$} \\
\hline G 305.200+0.019 & 4.1 & $0.9 \pm 6.2$ & $0.5 \pm 6.2$ & $7.2 \pm 3.0$ \\
G 305.202+0.208 & 4.1 & $-7.9 \pm 6.1$ & $-0.2 \pm 7.6$ & $10.8 \pm 4.4$ \\
\hline
\end{tabular}

data calibration. We assess that the main sources of error in our parallax measurements are from atmospheric effects which we are currently working to improve.

\section{PROPERTIES OF ASSOCIATED HMSFRS}

\subsection{Peculiar motion}

We used the $v_{\mathrm{lsr}}$ of the associated ${ }^{13} \mathrm{CO}$ molecular clump in Table 1 with the measured proper motions in Table 6 to determine the full 3D motion of G 305.200+0.019 and G 305.202+0.208 in the Galactic plane, which we present in Table 7 . The dynamical model of the Galaxy we use is derived from Reid et al. (2014) and assumes a flat rotation curve of the disc with a circular rotation speed of $\Theta_{0}=240 \mathrm{~km} \mathrm{~s}^{-1}$ at the radius of the Sun. The distance of the Sun from the Galactic Centre is taken to be $R_{0}=8.34 \mathrm{kpc}$ and the Solar Motion components are $U_{\odot}=10.70 \mathrm{~km} \mathrm{~s}^{-1}$ (towards the Galactic Centre), $V_{\odot}=15.60 \mathrm{~km} \mathrm{~s}^{-1}$ (clockwise and in the direction of Galactic rotation as viewed from the north Galactic pole) and $W_{\odot}=8.90 \mathrm{~km} \mathrm{~s}^{-1}$ (in the direction of the north Galactic pole). In Reid et al. (2014), there is a good fit to the model of spiral arm motions when an rms of about $5-7 \mathrm{~km} \mathrm{~s}^{-1}$ is assumed for each velocity component of HMSFR, which is reasonable for virial motions of stars in GMCs. Table 7 shows that at a distance of $4.1 \mathrm{kpc}$, the calculated peculiar velocity components for $\mathrm{G} 305.200+0.019$ and $G 305.202+0.208$ follow this trend inside the limits of uncertainty, though there appear to be deviations from the model for some components.

\subsection{Spiral arm allocation}

We have used Galactic CO emission measurements by Reid et al. (2016) and García et al. (2014) with the parallax distance in Table 6 to determine the spiral arm allocation of the $\mathrm{G} 305.2$ region. It appears that the region could either belong to the Centaurus or Carina spiral arms. In comparing the $v_{\mathrm{lsr}}$ of G $305.21+0.21$ (Table 1) with fig. 13 of Reid et al. (2016), the sources would favour the Centaurus spiral arm. However, their location at a Galactic longitude of $305^{\circ}$ places them a few degrees outside the tangent point of $\sim 310^{\circ}$. In terms of the Carina arm, there is a relatively large disparity of $\sim 15 \mathrm{~km} \mathrm{~s}^{-1}$ in the $v_{\text {lsr }}$.
We attempted to constrain the spiral arm association of G 305.2 by studying the modelled peculiar motions of G $305.200+0.019$ and $\mathrm{G} 305.202+0.208$ over a range of distances between 1 and $7 \mathrm{kpc}$. Our tests indicate reasonable peculiar motions expected for HMSFRS (Reid et al. 2009b) between 1 and 4 kpc (see Table 7), corresponding to the Carina spiral arm, and for distances $>5 \mathrm{kpc}$ corresponding to Centaurus. We concluded that the latter contradicts the parallax distance and exacerbates the longitude problem and therefore find strong evidence to associate the $\mathrm{G} 305.2$ region with the Carina-Sagittarius arm. This arm has been modelled by Sato et al. (2014) as a log-periodic spiral with a pitch angle of $\psi=19.8$ \pm 3.1 in the Galactocentric azimuth $(\beta)$ range $3.3<\beta<100.9$. We used the Bayesian Markov chain Monte Carlo procedure from Reid et al. (2014) to include the G 305.2 region to this model. In doing so, we increase the range of $\beta$ by $\sim 40^{\circ}$, to obtain an updated pitch angle of $\psi=19.0 \pm 2.6$ for the Carina-Sagittarius arm, now with greater confidence for the range $-34.8<\beta<100.9$.

Honig \& Reid (2015) show that the pitch angles of the arms of four nearby spirals typically fall within the range of $10^{\circ}<\psi<30^{\circ}$ and are highly scattered. This appears to be the case for arms in different galaxies, among different arms within a galaxy and also within individual spiral arms. We find our updated value of $\psi$ to be consistent when we model the arm over $-40^{\circ}<\beta<40^{\circ}$ and $-40^{\circ}<\beta<120^{\circ}$. This is consistent with Honig \& Reid (2015) who observe the stability of $\psi$ along arm segments over $5-10 \mathrm{kpc}$, which is applicable to our case.

\subsection{Source details}

In Fig. 6, we present the first VLBI maps of the 6.7$\mathrm{GHz}$ methanol maser emission towards G 305.200+0.019, G 305.202+0.208 and G 305.208+0.206. These have been produced from the 2013 November epoch of observations. Highresolution $\left(1.8 \times 1.4 \operatorname{arcsec}^{2}\right.$ beam size $)$ images of the $6.7-\mathrm{GHz}$ methanol masers in $\mathrm{G} 305.202+0.208$ have previously been made by Phillips et al. (1998), and Norris et al. (1993) and Phillips et al. have presented maps of the $6.7-\mathrm{GHz}$ methanol masers in $\mathrm{G} 305.208+0.206$. In producing the spot maps, we grouped the emission for each source which was present across contiguous spectral channels into a single maser feature. Each feature typically represents emission from between 4 and 20 spectral channels $\left(0.22-1.10 \mathrm{~km} \mathrm{~s}^{-1}\right)$ and the points in the figures are a flux densityweighted average in position and velocity. The methanol emission for these sources is measured to span between 190 and $1000 \mathrm{au}$, which is consistent with measurements from other VLBI observations of methanol maser emission (e.g. Bartkiewicz et al. 2008; Brunthaler et al. 2009). The errors in the relative positions between the features in each map are $<<1$ mas and the image noise is between 10 and $20 \mathrm{mJy}^{\text {beam }}{ }^{-1}$.

\section{$6.3 .1 G 305.200+0.019$}

We find the 6.7-GHz methanol maser emission associated with G 305.200+0.019 has eight distinct velocity components in Fig. 6 (top panel). The emission spans 0.50 arcsec in the north-south direction and 0.12 arcsec along the east-west direction. Except for the feature at $-31.96 \mathrm{~km} \mathrm{~s}^{-1}$, there is a velocity gradient in the emission with the seven features which form a simple and linear structure towards the north of the plot. This structure spans 0.06 arcsec (averaged along either axis), which corresponds to 245 au at a distance of $4.1 \mathrm{kpc}$ (from Table 6). The lone feature at $-37.07 \mathrm{~km} \mathrm{~s}^{-1}$ is offset 

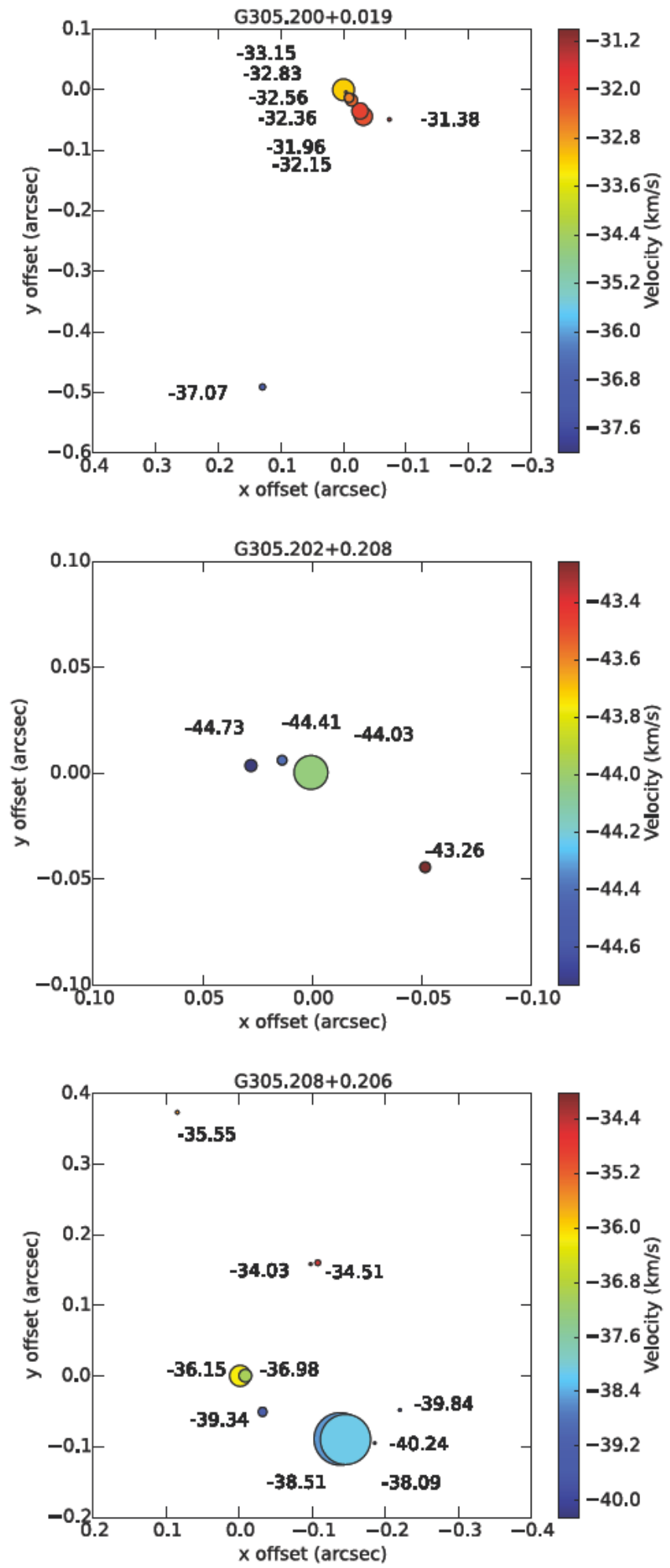

Figure 6. The 6.7-GHz methanol maser emission for G $305.200+0.019$ (top), G $305.202+0.208$ (middle) and G $305.208+0.206$ (bottom) from the 2013 November session. The numbers in the image field correspond to the flux density-weighted velocity of the associated feature. The size of the data points (relative to each source) are scaled to the integrated flux density of the strongest maser channel of the associated feature.
Table 8. Physical parameters of G $305.200+0.019$ from Hindson et al. (2012) adjusted to a preferred distance of $4.1 \mathrm{kpc}$.

\begin{tabular}{lcccc}
\hline $\begin{array}{l}n_{\mathrm{e}} \\
\left(\mathrm{cm}^{-3}\right)\end{array}$ & $\begin{array}{c}M_{\mathrm{H} \text { II }} \\
\left(\mathrm{M}_{\odot}\right)\end{array}$ & $\begin{array}{c}\log N_{L} \\
\left(\mathrm{~s}^{-1}\right)\end{array}$ & $\begin{array}{c}M_{*} \\
\left(\mathrm{M}_{\odot}\right)\end{array}$ & $\begin{array}{c}\text { Spectral } \\
\text { type }\end{array}$ \\
\hline $0.56 \times 10^{4}$ & $38.7 \times 10^{-4}$ & 46.19 & 14.9 & B 0.5 \\
\hline
\end{tabular}

from the centre of the image field by 0.51 arcsec. The maser spot which was used for parallax determination is associated with the feature with an integrated flux density of $30.0 \mathrm{Jy}$ at $-33.15 \mathrm{~km} \mathrm{~s}^{-1}$. In Fig. 2, we identify a feature at $-38.5 \mathrm{~km} \mathrm{~s}^{-1}$ for $\mathrm{G} 305.200+0.019$ which was undetected in Green et al. (2012). We were not able to identify this feature from our $1.2 \operatorname{arcsec}^{2}$ image cube and conclude that it is either resolved on VLBI baselines or may be associated with G 305.199+0.005.

Hindson et al. (2012) derive the properties of the embedded high-mass star for the candidate UC HII region associated with G $305.200+0.019$ and classify it as a B 1 source. Using their results along with the equations from Panagia \& Walmsley (1978), we estimate the electron density $n_{\mathrm{e}}$, mass of ionized hydrogen $M_{\mathrm{H} \text { II }}$, the Lyman continuum photon flux $N_{\mathrm{L}}$ in Table 8. From this, we find the (lower limit) mass of the ionizing source responsible for the observed Lyman flux to be a B 0.5-type star.

Using the distance of $4.1 \mathrm{kpc}$ for the IRAS source identified by Walsh et al. (1997), we find that G $305.202+0.019$ has a luminosity of $L=28.3 \times 10^{4} \mathrm{~L}_{\odot}$. This corresponds to a source with an O 6-5.5 spectral type (Panagia 1973), which is in contrast to the classification in Table 8 . The disparity can be explained by highmass stars forming in clusters and the derived spectral type from the arcminute-resolution IRAS observations is likely to indicate the total luminosity of the cluster as if it were produced by a single high-mass source.

\section{3 .2 G $305.202+0.208$}

The middle panel of Fig. 6 shows that the emission associated with G $305.202+0.208$ consists of four features with a simple structure spanning 0.08 arcsec in the east-west direction, corresponding to $330 \mathrm{au}$ at a distance of $4.1 \mathrm{kpc}$ (from Table 6). While the structure seen here is similar to that in Phillips et al. (1998), it appears that some weak emission to the north-east and north-west has fallen below the detection limit of our observations or has been resolved by VLBI. However, the feature at $-43.26 \mathrm{~km} \mathrm{~s}^{-1}$ was not detected by Phillips et al. (1998). The maser spot, which was used for parallax determination, is associated with the feature with an integrated flux density of $22.5 \mathrm{Jy}$ at $-44.03 \mathrm{~km} \mathrm{~s}^{-1}$.

Walsh et al. (1997) derive a luminosity of $20.9 \times 10^{4} \mathrm{~L}_{\odot}$ for the IRAS source associated with G 305.202+0.208. (Walsh et al. 1999 show that the IR emission is associated with G $305.202+0.208$ and not G 305.208+0.206, as presented in Walsh et al. 1997.) Using the updated distance of $4.1 \mathrm{kpc}$ to G $305.202+0.208$, we find that the luminosity is $16.6 \times 10^{4} \mathrm{~L}_{\odot}$, which would correspond to an $\mathrm{O} 6.5$ spectral source, if solitary, but is likely to be indicative of the cluster luminosity.

\section{$6.3 .3 G 305.208+0.206$}

G 305.208+0.206 shows the most complex distribution of emission of the sources in Fig. 6 (bottom panel), consisting of 10 features. The main cluster forms a ring-like structure with a diameter of $\sim 0.22$ arcsec, corresponding to 900 au at a distance of $4.1 \mathrm{kpc}$ 
(from Table 6). There is also a feature at $-35.55 \mathrm{~km} \mathrm{~s}^{-1}$ offset to the north-west by 0.38 arcsec from the centre of the image field. The emission does not appear to follow a simple or clear velocity gradient; however, there is a general decrease in velocity towards the south. Phillips et al. (1998) identify the strongest feature of this source as a single spot; however, we have resolved the emission into two features at -38.51 and $-38.09 \mathrm{~km} \mathrm{~s}^{-1}$. There is also weak emission at $-46 \mathrm{~km} \mathrm{~s}^{-1}$ in Phillips et al. (1998), in the region between the -36.15 and $-34.04 \mathrm{~km} \mathrm{~s}^{-1}$ features which is below the detection limit of our observations. The integrated flux density of the peak feature at $38.51 \mathrm{~km} \mathrm{~s}^{-1}$ is $122.3 \mathrm{Jy}$.

\section{CONCLUSION}

We have obtained parallaxes for methanol masers associated with the G 305.2 region. The parallax of G $305.200+0.019$ is measured to be $0.21 \pm 0.06$ mas and the parallax of $\mathrm{G} 305.202+0.208$ is measured to be $0.42 \pm 0.13$ mas. We combine these to obtain a variance-weighted average parallax of $0.25 \pm 0.05$ mas, corresponding to a distance of $4.1_{-0.7}^{+1.2} \mathrm{kpc}$ to the $\mathrm{G} 305.2$ region. We find the (near-) kinematic distance, using the latest Galactic parameters, to the ${ }^{13} \mathrm{CO}$ molecular clump associated with these masers to be $4.3_{-1.4}^{+2.2} \mathrm{kpc}$. While not inconsistent with the parallax distance, the kinematic distance has a larger uncertainty and provides evidence of the unreliability of this technique on providing distances for the study of HMSF.

VLBI observations of maser emission associated with HMSFRs are providing insights into the spiral structure of the Milky Way Galaxy. These measurements are predominantly from Northern hemisphere observations and do not probe the Galactic structure in the fourth quadrant. The LBA is the only VLBI instrument in the Southern hemisphere which is currently providing parallax distances to methanol maser sources in this region. Our LBA parallax results from G $305.200+0.019$ and G $305.202+0.208$ allow us to place the G 305.2 region in the Carina-Sagittarius arm, thereby extending the Galactic azimuth range of the sources in this arm by $\sim 40^{\circ}$. From this, we have revised the pitch angle of the CarinaSagittarius arm to $\psi=19.0 \pm 2.6$. The broader azimuth range allows the pitch angle to be determined with greater confidence, demonstrating the role of Southern hemisphere observations in determining the spiral structure of the Milky Way.

\section{ACKNOWLEDGEMENTS}

The LBA is part of the Australia Telescope National Facility which is funded by the Australian Government for operation as a National Facility managed by CSIRO and the University of Tasmania. We thank the referee (Dr Anita Richards) for helpful comments in reviewing this paper.

\section{REFERENCES}

Bartkiewicz A., Brunthaler A., Szymczak M., van Langevelde H. J., Reid M. J., 2008, A\&A, 490, 787

Beasley A. J., Conway J. E., 1995, in Zensus J. A., Diamond P. J., Napier P. J., eds., ASP Conf. Ser. Vol. 82, Very Long Baseline Interferometry and the VLBA. Astron. Soc. Pac., San Francisco, p. 327

Breen S. L., Ellingsen S. P., Caswell J. L., Green J. A., Voronkov M. A., Fuller G. A., Quinn L. J., Avison A., 2012, MNRAS, 421, 1703

Breen S. L., Ellingsen S. P., Contreras Y., Green J. A., Caswell J. L., Stevens J. B., Dawson J. R., Voronkov M. A., 2013, MNRAS, 435, 524

Brunthaler A., Reid M. J., Menten K. M., Zheng X. W., Moscadelli L., Xu Y., 2009, ApJ, 693, 424
Caswell J. L., Haynes R. F., 1987, A\&A, 171, 261

Caswell J. L., Vaile R. A., Ellingsen S. P., Whiteoak J. B., Norris R. P., 1995a, MNRAS, 272, 96

Caswell J. L., Vaile R. A., Forster J. R., 1995b, MNRAS, 277, 210

Clark J. S., Porter J. M., 2004, A\&A, 427, 839

Csengeri T. et al., 2014, A\&A, 565, A75

Cyganowski C. J., Brogan C. L., Hunter T. R., Churchwell E., 2009, ApJ, 702,1615

Danks A. C., Dennefeld M., Wamsteker W., Shaver P. A., 1983, A\&A, 118, 301

Davies B. et al., 2012, MNRAS, 419, 1871

De Buizer J. M., 2003, MNRAS, 341, 277

Deller A. T. et al., 2011, PASP, 123, 275

Ellingsen S. P., 2006, ApJ, 638, 241

Faimali A. et al., 2012, MNRAS, 426, 402

García P., Bronfman L., Nyman L.-Å., Dame T. M., Luna A., 2014, ApJS, 212, 2

Goddi C., Moscadelli L., Sanna A., 2011, A\&A, 535, L8

Green J. A. et al., 2012, MNRAS, 420, 3108

Greisen E. W., 2003, in Heck A., ed., Astrophysics and Space Science Library, Vol. 285, Information Handling in Astronomy - Historical Vistas. Kluwer, Dordrecht, p. 109

Hill T., Burton M. G., Minier V., Thompson M. A., Walsh A. J., HuntCunningham M., Garay G., 2005, MNRAS, 363, 405

Hindson L., Thompson M. A., Urquhart J. S., Clark J. S., Davies B., 2010 , MNRAS, 408, 1438

Hindson L., Thompson M. A., Urquhart J. S., Faimali A., Clark J. S., Davies B., 2012, MNRAS, 421, 3418

Hindson L., Thompson M. A., Urquhart J. S., Faimali A., Johnston-Hollitt M., Clark J. S., Davies B., 2013, MNRAS, 435, 2003

Honig Z. N., Reid M. J., 2015, ApJ, 800, 53

Krishnan V. et al., 2015, ApJ, 805, 129

McConnell D., Sadler E. M., Murphy T., Ekers R. D., 2012, MNRAS, 422, 1527

Ma C. et al., 2009, The Second Realization of the International Celestial Reference Frame by Very Long Baseline Interferometry. IERS Technical Note 35, International Earth Rotation and Reference System Service (IERS), International VLBI Service for Geodesy and Astrometry (IVS)

Menten K., 1991, in Haschick A. D., Ho P. T. P., eds., ASP Conf. Ser. Vol. 16 , Atoms, Ions and Molecules: New Results in Spectral Line Astrophysics. Astron. Soc. Pac., San Francisco, p. 119

Mioduszewski A. J., Kogan L., 2009, Strategy for Removing Tropospheric and Clock Errors using delzn Version 2.0. AIPS Memo 110, National Radio Astronomy Observatory and Cornell University

Moscadelli L., Goddi C., 2014, A\&A, 566, A150

Müller H. S. P., Menten K. M., Mäder H., 2004, A\&A, 428, 1019

Murphy T. et al., 2010, MNRAS, 402, 2403

Norris R. P., Whiteoak J. B., Caswell J. L., Wieringa M. H., Gough R. G., 1993, ApJ, 412, 222

Panagia N., 1973, AJ, 78, 929

Panagia N., Walmsley C. M., 1978, A\&A, 70, 411

Petrov L., Phillips C., Bertarini A., Murphy T., Sadler E. M., 2011, MNRAS, 414, 2528

Phillips C. J., Norris R. P., Ellingsen S. P., McCulloch P. M., 1998, MNRAS, 300,1131

Reid M. J., Honma M., 2014, ARA\&A, 52, 339

Reid M. J., Menten K. M., Brunthaler A., Zheng X. W., Moscadelli L., Xu Y., 2009a, ApJ, 693, 397

Reid M. J. et al., 2009b, ApJ, 700, 137

Reid M. J. et al., 2014, ApJ, 783, 130

Reid M. J., Dame T. M., Menten K. M., Brunthaler A., 2016, ApJ, 823,77

Russeil D., Georgelin Y. M., Amram P., Gach J. L., Georgelin Y. P., Marcelin M., 1998, A\&AS, 130, 119

Sanna A., Moscadelli L., Cesaroni R., Tarchi A., Furuya R. S., Goddi C. 2010, A\&A, 78, 23

Sato M. et al., 2014, ApJ, 793, 72

Sugiyama K. et al., 2014, A\&A, 562, A82 
Sutton E. C., Sobolev A. M., Ellingsen S. P., Cragg D. M., Mehringer D. M., Ostrovskii A. B., Godfrey P. D., 2001, ApJ, 554, 173

Voronkov M. A., Brooks K. J., Sobolev A. M., Ellingsen S. P., Ostrovskii A. B., Caswell J. L., 2006, MNRAS, 373, 411

Voronkov M. A., Caswell J. L., Ellingsen S. P., Sobolev A. M., 2010, MNRAS, 405, 2471

Walker C., Chatterjee S., 1999, Ionospheric Corrections Using GPS Based Models. VLBA Scientific Memo 23, National Radio Astronomy Observatory and Cornell University

Walsh A. J., Burton M. G., 2006, MNRAS, 365, 321

Walsh A. J., Hyland A. R., Robinson G., Burton M. G., 1997, MNRAS, 291, 261
Walsh A. J., Burton M. G., Hyland A. R., Robinson G., 1999, MNRAS, 309, 905

Walsh A. J., Bertoldi F., Burton M. G., Nikola T., 2001, MNRAS, 326, 36 Walsh A. J., Lee J.-K., Burton M. G., 2002, MNRAS, 329, 475

Walsh A. J., Chapman J. F., Burton M. G., Wardle M., Millar T. J., 2007, MNRAS, 380, 1703

Xu Y., Reid M. J., Menten K. M., Brunthaler A., Zheng X. W., Moscadelli L., 2009, ApJ, 693, 413

This paper has been typeset from a $\mathrm{T}_{\mathrm{E}} \mathrm{X} / \mathrm{LAT}_{\mathrm{E}} \mathrm{X}$ file prepared by the author. 